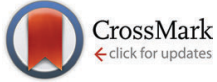

Cite this: Phys. Chem. Chem. Phys., 2016, 18, 20583

Received 6th May 2016 Accepted 23rd June 2016

DOI: $10.1039 / c 6 c p 03042 d$

www.rsc.org/pccp

\title{
Electrical impedance spectroscopy of a PET chip sandwiched between two disk electrodes: understanding the contribution of the polymer/electrode interface
}

\author{
M. Kechadi, ${ }^{a b}$ L. Chaal, $^{b}$ V. Vivier, ${ }^{a}$ B. Tribollet ${ }^{a}$ and J. Gamby $\dagger^{\star a}$
}

\begin{abstract}
This work is devoted to the understanding of the dielectric impedance response of a semi-crystalline polyethylene terephthalate (PET) membrane sandwiched between two disk electrodes under alternate voltage excitation in the frequency range between $1 \mathrm{MHz}$ and $25 \mathrm{mHz}$. Experimental results obtained for various PET thicknesses (36, 50 and $100 \mu \mathrm{m})$ highlighted the influence of the contact resistance at the electrode/polymer interface. For a better understanding of the PET/electrode interface behaviour, the experiments were compared with simulations performed for three different descriptions: the direct use of electrical equivalent circuits, an analytical model accounting for a power-law distribution of resistivity, and a numerical model (finite element simulations of the whole cell). The results highlight that the resistivity distribution obtained using the power-law model provided an appropriate description of the system in the frequency range investigated while the use of the CPE model is only consistent for low-frequencies (below $1 \mathrm{~Hz}$ ).
\end{abstract}

\section{Introduction}

Polyethylene terephthalate (PET) is one of the most widely used insulating polymers due to its excellent electrical, thermal and mechanical properties in high-performance foil capacitors. The investigation of the dielectric properties of a polymer such as PET is very important for both fundamental and industrial approaches because it allows the efficiency of its electrical resistance for insulation to be checked. Indeed, the dielectric properties of PET or chemically or physically modified PET remain crucial for many kinds of applications. Reddish et al. ${ }^{1}$ pioneered the fundamental description of PET dielectric property measurements and calculations. In brief, they studied several PET crystallinity degrees for frequencies ranging from $100 \mathrm{~Hz}$ to $10 \mathrm{MHz}$ and for temperatures between $-80^{\circ} \mathrm{C}$ and $180^{\circ} \mathrm{C}$. They identified dielectric transitions depending on the crystallinity degree: the first one at $100{ }^{\circ} \mathrm{C}$ was ascribed to dipole motion inside the monomer, whereas the second one at $-50{ }^{\circ} \mathrm{C}$ was associated with the thermal motion of $\mathrm{OH}$ groups in amorphous phases. Moreover, for the low frequency (LF) and high temperature (HT) domains, they evidenced a dc-conductive

\footnotetext{
${ }^{a}$ Sorbonne Universités, UPMC Univ Paris 06, CNRS, Laboratoire Interfaces et Systèmes Electrochimiques, 4 place Jussieu, F-75005, Paris, France. E-mail: Jean.gamby@upmc.fr

${ }^{b}$ Laboratoire d'Electrochimie, Corrosion et de Valorisation Energétique (LECVE), Faculté de Technologie, Université A. MIRA - Béjaia, 06000, Algeria

$\dagger$ Present address: Centre de Nanosciences et de Nanotechnologies, CNRS, Univ. Paris-Sud, C2N - Marcoussis, 91460 Marcoussis, France. E-mail: jean.gamby@lpn.cnrs.fr
}

contribution ascribed to the charge conduction through the PET. In continuity of Reddish's work, Maxwell ${ }^{2}$ and $\mathrm{Neagu}^{3}$ focused their investigations on the origin of the relaxation process through the PET membrane. This was achieved by comparing the dynamic mechanical and dielectric results obtained on amorphous PET-additive blends using different techniques such as NMR and dielectric relaxation spectroscopy (DRS). They concluded that local molecular movements of carbonyl dipole groups corresponded to low temperature peaks in the frequency range from $10 \mathrm{kHz}$ to $1 \mathrm{~Hz}$ while the high temperature motions were attributed to the nonpolar phenyl rings. Neagu et al. ${ }^{3,4}$ performed DRS experiments on $6 \mu \mathrm{m}$ thick films with $68 \%$ degree of crystallinity. It was demonstrated that PET showed a primary dielectric $\alpha$-relaxation linked to the glass transition of the crystalline regions of the polymer and a secondary $\beta$-relaxation, which accounts for the amorphous transitions. Those two relaxations explain the increase of the values of $\tan (\delta)$ and $\varepsilon^{\prime}$ at both high temperatures and high frequencies (i.e. above $140{ }^{\circ} \mathrm{C}$ and $10^{5} \mathrm{~Hz}$ ). High temperatures and low frequency regions (HT/LF) were studied within the complex permittivity formalism, which describes the high values of $\tan (\delta)$ and $\varepsilon^{\prime}$ (corresponding to the $\rho$ peak found in thermally stimulated depolarization current). ${ }^{5,6}$ In both cases, these properties evolve with temperature following Vogel-Tamman-Fulcher equations. ${ }^{7,8}$ These dielectric properties, which are a function of frequency and temperature, are thus essential for application of PET as a foil capacitor.

In recent years, due to the emergence of flexible microfluidic polymer systems for bioanalytical applications or patch integrating 
electrodes for rapid medical diagnostics, the understanding of the surrounding polymer impedance response has played an important role in interpreting the sensor response. ${ }^{9}$ In this context, impedance spectroscopy is a well-adapted technique not only for sensor or biosensor development but also for the characterization of non-conductive organic materials. ${ }^{10-12}$ This present work is devoted to the electrical behaviour of commercially available PET membranes of different thicknesses in the large frequency range. This paper constitutes the first part of our investigations for a better understanding of the role of the PET membrane in the global impedance through an electrode/PET macrodevice.

\section{Materials and methods}

\subsection{PET disk fabrication}

Three thicknesses $(d=36,50$ and $100 \mu \mathrm{m})$ of a $60 \%$ weight semi-crystalline PET membrane were perforated with a $5 \mathrm{~mm}$ diameter circular punch. In order to prevent high contact resistance at the PET/disk electrode interface, gold metallization of the PET facets was performed. A $10 \mathrm{~nm}$ thick layer of sputtered gold was deposited on PET disks using magnetron sputtering (PFEIFFER, PLS 500) in a purified argon atmosphere (2 Pa) and a power of $30 \mathrm{~W}$ for $12 \mathrm{~min}$. The macrodevice consisted of two stainless-steel tubular electrodes between which the golden-PET disk sample was sandwiched (Fig. 1a and b). A hollow-cylinder in Teflon was designed as a chip holder to avoid any parasitic current during measurement.

\subsection{Apparatus}

The electric impedance measurements were performed using a dielectric interface - gain phase impedance analyzer (Solartron 1296A) coupled with a frequency response analyser (FRA 1255A)
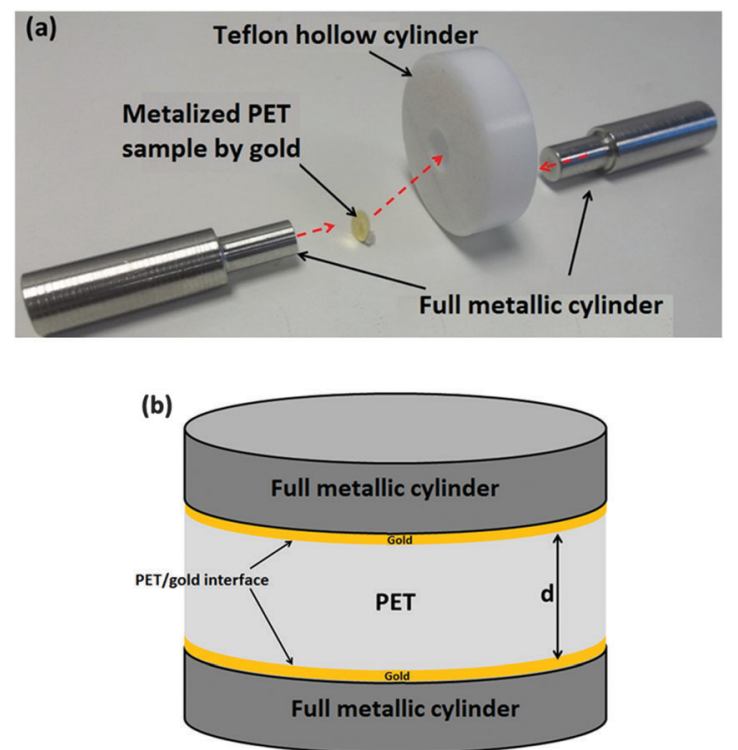

Fig. 1 (a) Chip holder in Teflon with two cylindrical stainless steal electrodes for measuring dielectric impedance spectra on the PET disk. (b) Schematic representation of the chip comprising the $\mathrm{PET/gold}$ interface. and computer-controlled with the Smart software (Solartron Analytical). The two electrodes were connected to a dielectric interface apparatus where the PET gold disk was inserted for test in the macrodevice as sketched in Fig. 1a. A linear relationship between ac-potential perturbation and current was checked in the whole frequency range for the ac amplitudes tested i.e. from $10 \mathrm{mV}$ to $1 \mathrm{~V}$. For further experiments, an ac-potential perturbation of $100 \mathrm{mV}$ amplitude was applied and data were collected in the frequency range from $1 \mathrm{MHz}$ to $25 \mathrm{mHz}$. In order to understand the dielectric impedance response of PET films, the modelling of the impedance using electrical equivalent circuits and the finite element method were performed in the frequency range from $1 \mathrm{MHz}$ to $1 \mathrm{mHz}$. In fact, the choice of a broader frequency range for the modelling allows predicting the PET behaviours in the very low frequency domain and thus a better description of the PET properties from the analysis of the impedance diagrams.

\section{Results and discussion}

\subsection{Modelling of the dielectric response of PET using electrical equivalent circuits}

The value of the geometric capacitance of the PET disk sandwiched between two electrodes was readily obtained by measuring the charging current between these electrodes, which was achieved by performing electric impedance measurements. ${ }^{13-15}$ The measured electric impedance through the PET disk of different
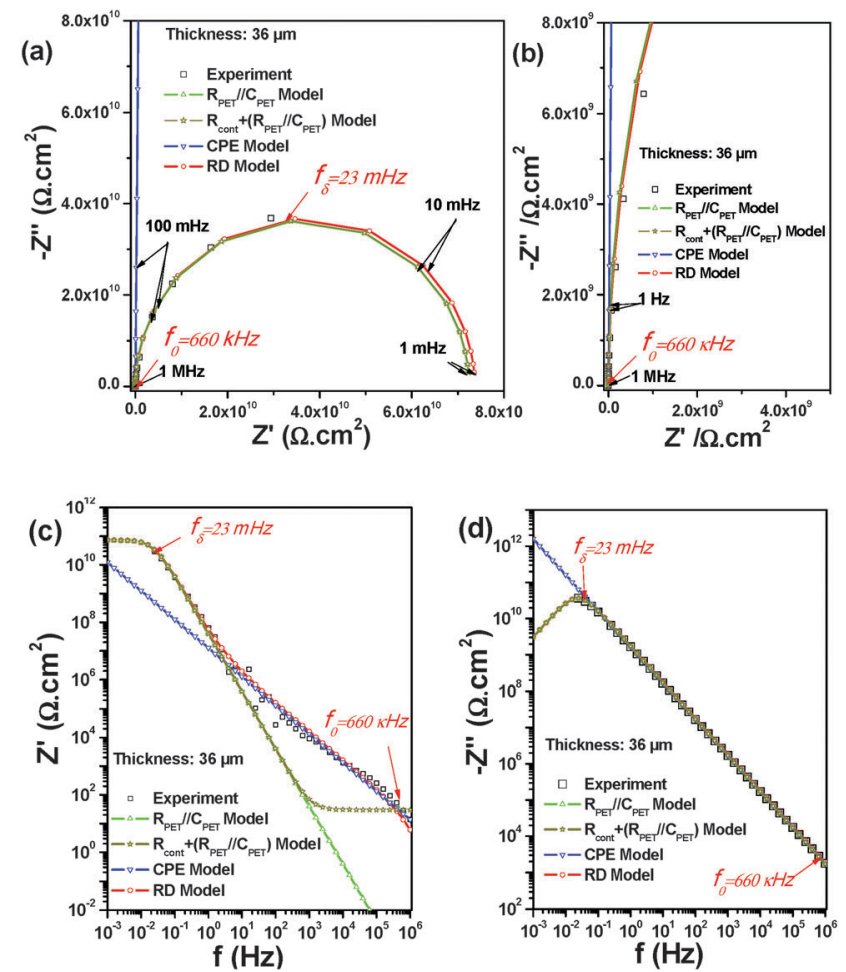

Fig. 2 Modeling using electrical equivalent circuits of impedance diagrams measured on a PET disk of thickness $36 \mu \mathrm{m}$. (a) and (b) Nyquist diagram. (c) Real part with frequency. (d) Imaginary part with frequency. 

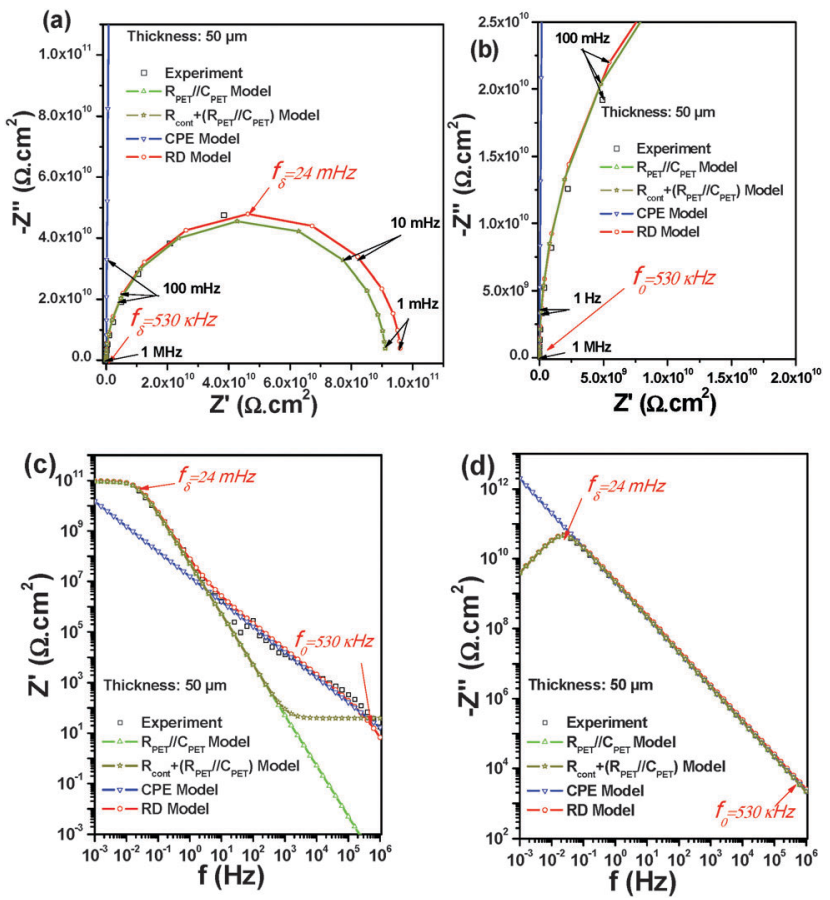

Fig. 3 Modelling using electrical equivalent circuits of impedance diagrams measured on a PET disk of thickness $50 \mu \mathrm{m}$. (a and b) Nyquist diagram. (c) Real part with frequency. (d) Imaginary part with frequency.

thicknesses (36, 50 and $100 \mu \mathrm{m})$ is shown in Fig. 2. In a first approach, data analysis was performed using electrical equivalent circuits. In the Nyquist representation (Fig. 2a, b, 3a, b and $4 \mathrm{a}, \mathrm{b})$ a $R / / C$ loop is observed in the low-frequency range whatever the PET thickness used. The $R$ element is ascribed to the PET membrane resistance, while the $C$ element accounts for the PET membrane capacitance between the two disk electrodes as defined in eqn (1), in which the relative permittivity $\left(\varepsilon_{\mathrm{r}, \mathrm{PET}}\right)$ and the sample thickness $\left(d_{\mathrm{PET}}\right)$ were taken into account. As expected, it can be observed that the loop diameter, related to the PET resistance, increases when the PET thickness increases. The real part of the measured impedance depends on the applied frequency (Fig. 2c, 3c and 4c). Indeed, in high frequencies $(1 \mathrm{MHz})$ the real part is about $50 \Omega \mathrm{cm}^{2}$ while it reaches $10^{11} \Omega \mathrm{cm}^{2}$ in low frequencies $(10 \mathrm{mHz})$. Whatever the PET thickness used, a capacitive behaviour is observed between $1 \mathrm{MHz}$ and $1 \mathrm{~Hz}$ (Fig. 2d, 3d and 4d), which is characterized by a slope of 0.99 obtained in the representation of the imaginary part of the impedance as a function of frequency. As mentioned above, the theoretical value of the PET membrane capacitance can be estimated as:

$$
C_{\mathrm{PET}, \text { theo }}=\frac{\varepsilon_{0} \varepsilon_{\mathrm{r}, \mathrm{PET}}}{d_{\mathrm{PET}}}
$$

where $d_{\mathrm{PET}}$ is the PET thickness $(\mathrm{m}), \varepsilon_{0}$ is the vacuum permittivity $\left(8.85 \times 10^{-12} \mathrm{~F} \mathrm{~m}^{-1}\right)$, and $\varepsilon_{\mathrm{r}, \mathrm{PET}}$ is the relative permittivity of PET (see Section 3.4).

Using eqn (1), the theoretical values of PET capacitance are found to be equal to $8.3 \times 10^{-11}, 6 \times 10^{-11}$ and $3 \times 10^{-11} \mathrm{~F} \mathrm{~m}^{-2}$ for PET thicknesses of 36, 50 and $100 \mu \mathrm{m}$, respectively.
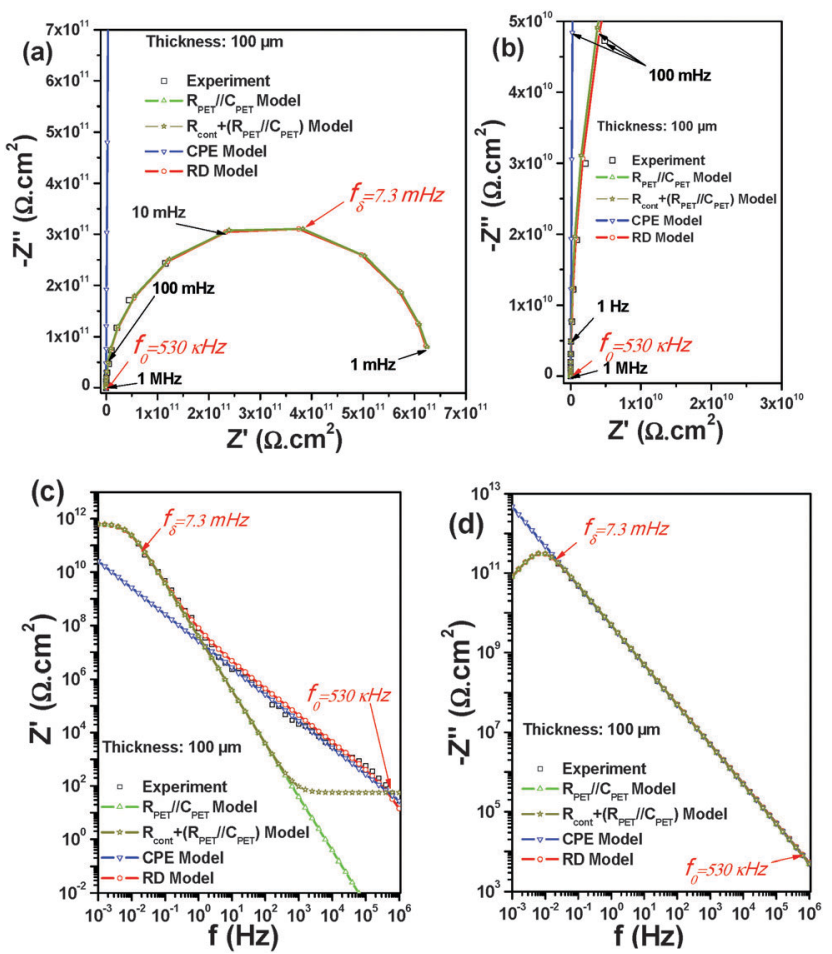

Fig. 4 Modeling using electrical equivalent circuits of impedance diagrams measured on a PET disk of thickness $100 \mu \mathrm{m}$. (a and b) Nyquist diagram. (c) Real part with frequency. (d) Imaginary part with frequency.

Moreover, the experimental value of PET capacitance can be graphically estimated using eqn (2).

$$
C_{\text {PET }, \exp }=\frac{1}{2 \pi f\left(-Z^{\prime \prime}\right)}
$$

where $f$ is the frequency in $\mathrm{Hz}$ and $Z^{\prime \prime}$ is the imaginary part of the impedance in $\Omega \mathrm{cm}^{2}$.

The experimental values obtained for capacitance are equal to $9.3 \times 10^{-11}, 7.2 \times 10^{-11}$ and $3.2 \times 10^{-11} \mathrm{~F} \mathrm{~cm}^{-2}$ for PET thicknesses of 36, 50 and $100 \mu \mathrm{m}$, respectively. These values of $C_{\mathrm{PET}, \exp }$ are very close to those of $C_{\mathrm{PET} \text {,theo }}$ previously determined, particularly for the $100 \mu \mathrm{m}$ PET thickness.

For a better understanding of the dielectric behaviour of PET, particularly for the real part of impedance, the modelling of the results was carried out using the electrical circuit model and the finite element method. As mentioned above, the dielectric membrane sandwiched between the two disk electrodes can be represented by a resistance $R_{\mathrm{PET}}$ and a geometric capacitance $C_{\mathrm{PET}}$. Traditionally, the dielectric response of a polymer membrane is represented by a parallel association $R_{\mathrm{PET}} / / C_{\mathrm{PET}}{ }^{16}$ The results obtained after the fitting procedure of the experimental impedance diagrams with the latter circuit are presented in Fig. 2-4 for the 36, 50, and $100 \mu \mathrm{m}$ PET membrane thicknesses, respectively. The values obtained from the fitting of the imaginary part of the impedance are in good agreement with the geometric capacitances previously estimated from eqn (1). Concerning the real part of the measured impedance, the simulated data in the low-frequency domain allowed the PET resistance estimation that 
Table 1 Parameter model of the $R_{\mathrm{PET}} / / C_{\mathrm{PET}}$ circuit from $1 \mathrm{MHz}$ to $1 \mathrm{mHz}$. The values of the resulting $\chi^{2}$ statistic were obtained with errors of fitted values, $\sigma=0.01$, for frequency ranges

\begin{tabular}{llll}
\hline$d(\mu \mathrm{m})$ & $R_{\mathrm{PET}}\left(\times 10^{10} \Omega\right)$ & $C_{\mathrm{PET}}\left(\times 10^{-11} \mathrm{~F} \mathrm{~cm}^{-2}\right)$ & $\chi^{2}(\%)$ \\
\hline 36 & 7.3 & 9.3 & 2.47 \\
50 & 9 & 7.2 & 2.12 \\
100 & 63 & 3.2 & 1.90
\end{tabular}

corresponds to the loop diameter of the Nyquist plots (Fig. 2a, b, $3 \mathrm{a}, \mathrm{b}$ and $4 \mathrm{a}, \mathrm{b}$ ). As listed in Table 1, high PET resistance values are found to be equal to $7.3 \times 10^{10}, 9 \times 10^{10}$ and $6.3 \times 10^{11} \Omega \mathrm{cm}^{2}$ for 36, 50, and $100 \mu \mathrm{m}$, respectively. However, as shown in Fig. 2c, $3 \mathrm{c}$ and $4 \mathrm{c}$, a significant difference between the simulated plots and the experimental plots is observed in the high-frequency domain. This difference is probably due to an interfacial effect or to a bulk effect due to the heterogeneity of the PET material.

Assuming an interfacial effect, a contact resistance effect, $R_{\text {cont }}$, was taken into account in series in the model $R_{\mathrm{PET}} / / C_{\mathrm{PET}}$ circuit. The simulated results obtained using the $R_{\text {cont }}+\left(R_{\mathrm{PET}} / /\right.$ $\left.C_{\mathrm{PET}}\right)$ model are presented in Fig. 2-4, and the corresponding parameters are listed in Table 2 . The obtained values of 30,39 and $56 \Omega \mathrm{cm}^{2}$ were found to be dependent on the PET thickness. In fact, as observed in Fig. 2, the plateau corresponding to the contact resistance was not observed in the high frequency domain and the experimental $R_{\text {cont }}$ value should be below $50 \Omega \mathrm{cm}^{2}$. However, whatever the $R_{\text {cont }}$ value, the $R_{\text {cont }}+\left(R_{\mathrm{PET}} / / C_{\mathrm{PET}}\right)$ model is not appropriate to account for the experimental data, because for the smaller value of $R_{\text {cont }}$ the $R_{\text {cont }}+\left(R_{\mathrm{PET}} / / C_{\mathrm{PET}}\right)$ model tends toward the $R_{\mathrm{PET}} / / C_{\mathrm{PET}}$ model.

Furthermore, the most significant difference between the experimental real part and the simulated one is in the intermediate frequency domain $(500 \mathrm{kHz}$ to $10 \mathrm{~Hz})$, whatever the PET thickness. At this stage, the modified electrical circuit model $R_{\text {cont }}+\left(R_{\mathrm{PET}} / / C_{\mathrm{PET}}\right)$ does not represent the behaviour observed.

The constant phase element (CPE) model was abundantly used to simulate the observed distribution in heterogeneous materials. In some cases, the structure is essentially represented by a CPE in parallel with a resistance. ${ }^{17}$ Recent studies have proposed a physical interpretation of the CPE behaviour. ${ }^{18,19}$ In brief, the CPE behaviour is mainly due to the distribution of the $R / / C$ time constant and cannot be explained by the variation in the relative permittivity. ${ }^{18,20}$ The CPE model was thus used to simulate our results. In a first view, a good agreement between experiment and fits is observed for frequencies between $1 \mathrm{MHz}$ and $1 \mathrm{~Hz}$ (Fig. 2-4). The value of CPE element, $Q_{\mathrm{CPE}}$, for each thickness was found to be close to the geometric capacitance and the fitted value of the CPE exponent is equal to the experimental one which was

Table 2 Parameter model of the $R_{\text {cont }}+\left(R_{\mathrm{PET}} / / C_{\mathrm{PET}}\right)$ circuit from $1 \mathrm{MHz}$ to $1 \mathrm{mHz}$. The values of the resulting $\chi^{2}$ statistic were obtained with errors of fitted values, $\sigma=0.01$, for frequency ranges

\begin{tabular}{lllll}
\hline$d(\mu \mathrm{m})$ & $R_{\text {cont }}\left(\Omega \mathrm{cm}^{2}\right)$ & $R_{\mathrm{PET}}\left(\times 10^{10} \Omega \mathrm{cm}^{2}\right)$ & $C_{\mathrm{PET}}\left(\times 10^{-11} \mathrm{~F} \mathrm{~cm}^{-2}\right)$ & $\chi^{2}(\%)$ \\
\hline 36 & 30 & 7.3 & 9.3 & 2.45 \\
50 & 39 & 9 & 7.3 & 2.05 \\
100 & 56 & 63 & 3.2 & 1.91
\end{tabular}

Table 3 Parameter CPE model from $1 \mathrm{MHz}$ to $1 \mathrm{~Hz}$. The values of the resulting $\chi^{2}$ statistic were obtained with errors of fitted values, $\sigma=0.01$, for frequency ranges

\begin{tabular}{llcc}
\hline$d(\mu \mathrm{m})$ & $Q_{\mathrm{CPE}}\left(\times 10^{-11} \Omega \mathrm{s}^{\alpha} \mathrm{cm}^{-2}\right)$ & $\alpha_{\mathrm{CPE}}$ & $\chi^{2}(\%)$ \\
\hline 36 & 9.6 & 0.99 & 12.9 \\
50 & 7.6 & & 6.97 \\
100 & 3.3 & & 4.39
\end{tabular}

graphically obtained through the slope of the imaginary part as a function of the frequency i.e. $\alpha_{\mathrm{CPE}}=0.99$. However, for frequencies lower than $1 \mathrm{~Hz}$, the CPE model does not represent the experimental results, especially for the real part of impedance. The Nyquist plots presented in Fig. 2-4 with a CPE exponent close to 1 highlight that this model is valid for a restricted frequency domain, only. Thus, the parameters listed in Table 3 were obtained for a frequency window restricted from $1 \mathrm{MHz}$ to $1 \mathrm{~Hz}$ in order to obtain more accurate values.

\subsection{Modelling of dielectric response of PET using the analytical model}

Let us consider now the second hypothesis, which consists of ascribing the observed change in the real part of impedance to a bulk effect in the PET material to resistivity distribution. ${ }^{20}$ Historically, this resistivity distribution model was introduced to simulate the change in material resistance such as aluminium oxides or stainless steel along its thickness. ${ }^{18,20,21}$ In brief, the resistivity distribution ( $\mathrm{RD}$ ) model was proposed to explain the origin of the constant phase element (CPE) behaviour. It can be seen as a series of $n R / / C$ elements ${ }^{20}$ as sketched in Fig. 5. This RD model can be extended for modelling the non-uniform variation observed for the resistive component of the dielectric impedance in a semi-crystalline polymer such as PET. ${ }^{20}$

For this purpose, a unidirectional variation of resistance in the bulk of PET is assumed as follows:

$$
\frac{\rho}{\rho_{\delta}}=\xi^{-\gamma}
$$

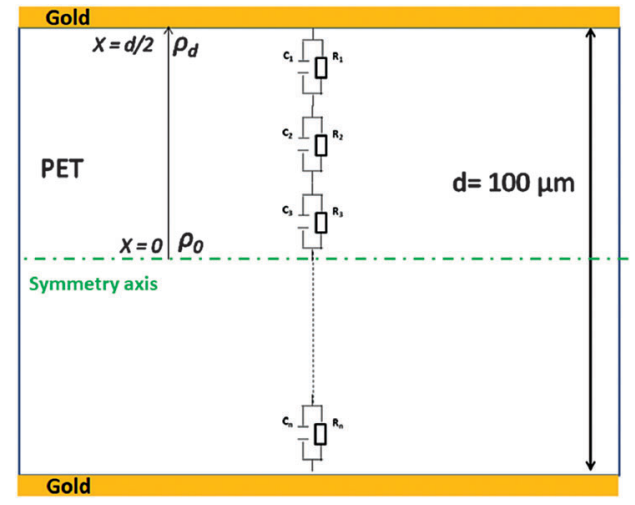

Fig. 5 A schematic representation of the resistivity distribution using the Voigt circuit in the PET disk with thickness $(d)$. Symmetry axis $(x=0)$ represents the bulk resistivity $\rho_{0}$ and the global impedance of the PET film is given by the double of estimated impedance using the RD model. 
with

$$
\xi=\frac{x}{\delta}
$$

where $\rho_{\delta}(\Omega \mathrm{cm})$ is the PET resistivity at the interface $x=\delta, \rho_{0}$ $(\Omega \mathrm{cm})$ is the PET resistivity of the bulk PET film $(x=0), \xi$ is the normalised distance, and $\delta$ is the PET thickness.

Then, the expression of $\rho / \rho_{\delta}=f(\xi)$ can be rewritten as:

$$
\frac{\rho}{\rho_{\delta}}=\left(\frac{\rho_{\delta}}{\rho_{0}}+\left(1-\frac{\rho_{\delta}}{\rho_{0}}\right) \xi^{\gamma}\right)^{-1}
$$

The impedance of distributed resistivity along the thickness $\delta$, $Z_{\mathrm{RD}}(\omega)$, is defined as follows

$$
Z_{\mathrm{RD}}(\omega)=\int_{0}^{\delta} \frac{\rho(x)}{1+j \omega \varepsilon_{0} \varepsilon \rho(x)} \mathrm{d} x
$$

The latter can be expressed as

$$
Z_{\mathrm{RD}}(\omega)=\delta \int_{0}^{1} \frac{1}{a(\omega)+b \xi \gamma} \mathrm{d} \xi
$$

where

$$
\begin{aligned}
& a(\omega)=\rho_{0}{ }^{-1}+j \omega \varepsilon_{0} \varepsilon \\
& b(\omega)=\rho_{\delta}{ }^{-1}-\rho_{0}{ }^{-1}
\end{aligned}
$$

and

$$
\alpha=\frac{\gamma-1}{\gamma}
$$

In the integral equation of the distributed impedance, two characteristic frequencies $f_{0}=\left(2 \pi \rho_{0}\right)^{-1}$ and $f_{\delta}=\left(2 \pi \rho_{\delta}\right)^{-1}$ can be defined from $\rho_{0}$ and $\rho_{\delta}$, respectively. The simulated results of the experimental data using the $\mathrm{RD}$ model are shown in Fig. 2-4. Conversely to the previous models, the simulated real parts of impedance superimpose well with the experimental one over the whole frequency range investigated experimentally (Fig. 2c, 3c and 4c). This indicates that the RD model is a better description to simulate the resistive component variation observed with the PET membrane. The obtained values for parameters with the RD model are listed in Table 4. A significant difference between the resistivity at the interface and in the bulk PET is highlighted. Indeed, the resistivity varies form $10^{6}(\Omega \mathrm{cm})$ at the interface to $10^{13}(\Omega \mathrm{cm})$ in the bulk PET. It is interesting to underline that the value of $\rho_{0}$ found is to be in agreement with the value given by the supplier $\left(10^{14} \Omega \mathrm{cm}\right)$. As shown in Table 4 , the $\gamma$ parameter is about 180, leading to an $\alpha$ value close to $1(\approx 0.99)$ using eqn $(10)$, which confirms the quasi-ideal capacitive behaviour of the PET membrane. Finally,

Table 4 Parameter resistivity distribution model (RD) from $1 \mathrm{MHz}$ to $1 \mathrm{mHz}$. The values of the resulting $\chi^{2}$ statistic were obtained with errors of fitted values, $\sigma=0.01$, for frequency ranges

\begin{tabular}{llllll}
\hline$d(\mu \mathrm{m})$ & $\rho_{\delta}\left(\times 10^{6} \Omega \mathrm{cm}\right)$ & $\rho_{0}\left(\times 10^{13} \Omega \mathrm{cm}\right)$ & $\gamma$ & $\alpha_{\mathrm{RD}}$ & $\chi^{2}(\%)$ \\
\hline 36 & 0.8 & 2.2 & 180 & 0.99 & 1.68 \\
50 & 1 & 2.2 & & & 1.55 \\
100 & 1 & 7 & & & 1
\end{tabular}

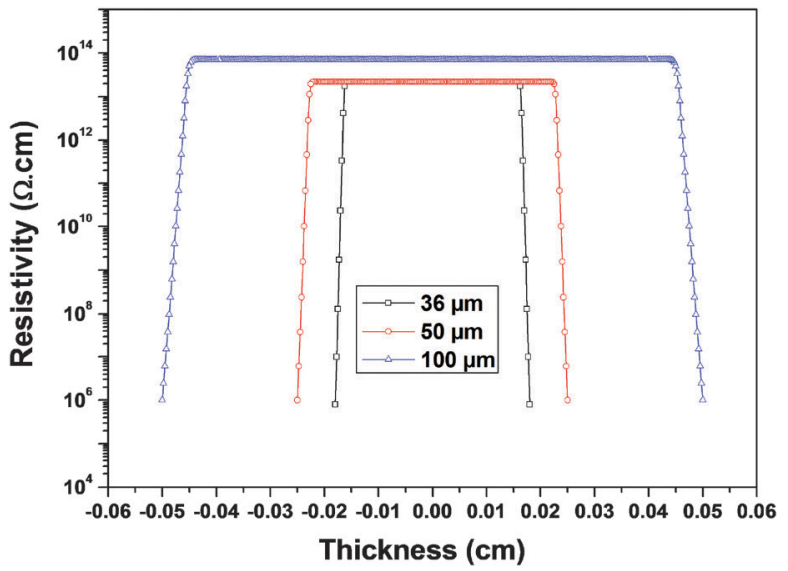

Fig. 6 Plots of resistivity variation along PET thickness.

the characteristic frequencies $f_{0}$ and $f_{\delta}$ are found to be $600 \mathrm{kHz}$ and $24 \mathrm{mHz}$, respectively. Nevertheless, it should be mentioned that the good simulated result of the experimental data using the RD model does not necessarily imply a physical meaning.

To confirm the validity of the RD model, the resistivity variation obtained from eqn (5) along the PET sample thickness is plotted, and the resistivity profiles are shown in Fig. 6. These profiles show that, whatever the sample thickness, the resistivity decreases abruptly at the edge of the PET layers and the variations are only located near the PET/gold interface. This result confirms the hypothesis that all the variations of resistivity take place at the interfaces, and are also in agreement with the pure capacitive behaviour of the PET membrane.

At this stage, taking into account the previous simulated results with the electrical circuit model $R_{\mathrm{cont}}+\left(R_{\mathrm{PET}} / / C_{\mathrm{PET}}\right)$, which has shown that the addition of a constant resistance at the PET interfaces does not describe the experimental results, it can be assumed that resistivity distribution is located on the edges of the PET membranes. Moreover, both the CPE model and the $\mathrm{RD}$ model lead to a good representation of the dielectric response of PET for frequencies between $1 \mathrm{MHz}$ and $1 \mathrm{~Hz}$. However, for frequencies lower than $1 \mathrm{~Hz}$ the CPE model does not represent the experimental results, in particular for the real parts of the impedance while the RD model is still valid in the full frequency range i.e. down to $25 \mathrm{mHz}$. These results confirm that the dielectric response of PET in high and medium frequencies can be represented by a CPE behaviour. From a physical point of view, this means that the resistivity distribution in PET occurs at the interface in a thin thickness layer. The choice of using one of these two models for representing the PET interface depends on the frequency range explored. In addition, one of the advantages of the CPE model in comparison with the $\mathrm{RD}$ model is to reduce the number of parameters to be considered.

\subsection{Modelling of the dielectric response of the PET using finite element simulation}

The modelling of the dielectric response of PET using the finite element was carried out using Comsol Multiphysics software. In our case, it consists of solving the Ampere-Maxwell equation 
in the three dimensions. The electrical properties of the PET sample which was used as the input in the model are the electrical conductivity $\left(\sigma_{\mathrm{PET}}\right.$ in $\left.\mathrm{S} \mathrm{m}^{-1}\right)$ and the relative permittivity $\left(\varepsilon_{\mathrm{PET}}\right)$. The latter is defined as a constant $\left(\varepsilon_{\mathrm{PET}}=3.4\right)^{22}$ in the frequency range between $1 \mathrm{MHz}$ and $25 \mathrm{mHz}$. Concerning the electrical conductivity, it may be considered as a constant parameter or frequency-dependent parameter. For the latter, it is expressed as $^{23-25}$

$$
\sigma(\omega)_{\mathrm{PET}}=\sigma_{\mathrm{dc}}+\sigma_{\mathrm{ac}}(\omega)
$$

where $\sigma_{\mathrm{dc}}$ is the electrical conductivity of PET in DC current and $\sigma_{\mathrm{ac}}$ is the electrical conductivity of PET in AC current.

The $\sigma_{\mathrm{ac}}$ conductivity was introduced by Jonscher, ${ }^{26,27}$ considering a power-law variation of conductivity as a function of frequency, as follows

$$
\sigma(\omega)_{\mathrm{PET}}=\sigma_{\mathrm{dc}}+A \omega^{s}
$$

where $s$ is the power-law exponent (between 0.5 and 1) reflecting the distribution and $A$ is a temperature-dependent parameter.

From a physical point of view, eqn (12) denotes that the dipole in the material with $W_{1}$ and $W_{2}$ energies and a jump time $(\tau)$ can be oriented in two or more different directions. This analysis was deduced from the established models of Debye and Frohlich. ${ }^{28,29}$ First, the global electric potential $\left(V_{t}\right)$ in the PET domain was set to $V_{t=0}=0$, and the boundary conditions were defined as an electrical insulation $(\mathrm{nJ}=0)$ with the external PET disk environment. At the PET/gold interfaces the applied voltages $V_{\mathrm{e} 1}$ and $V_{\mathrm{e} 2}$ were set to $+50 \mathrm{mV}$ and $-50 \mathrm{mV}$, respectively. If an interfacial impedance was considered, the condition of a distributed impedance was applied with an electrical conductivity that may be either a constant or a variable. To sum up, four cases were considered in this study that depend on the electrical conductivity $\left(\sigma_{\mathrm{PET}}\right)$ : (i) constant on the PET domain and on the PET interfaces, (ii) variable on the PET domain and constant on the interfaces, (iii) constant on the PET domain and variable on the interfaces and (iv) variable on the PET domain and on the interfaces. The meshing step on the geometry was performed by using a triangular mesh which permits us to obtain a weak convergence and an independent solution with the mesh dimension.

The results of the modelling of the dielectric behaviour of PET at $100 \mathrm{mV}$ are shown in Fig. 7-9. The modelling of the experimental spectra with a constant $\sigma_{\mathrm{PET}}$ led to set the bulk PET conductivity at $\sigma_{\mathrm{PET}}=1.5 \times 10^{-12} \mathrm{~S} \mathrm{~m}^{-1}$ for $100 \mu \mathrm{m}$ thickness and at $\sigma_{\mathrm{PET}}=4.7 \times 10^{-12} \mathrm{~S} \mathrm{~m}^{-1}$ for $36 \mu \mathrm{m}$ and $50 \mu \mathrm{m}$ thicknesses. In Nyquist diagrams presented in Fig. 7a, 8a and 9a, a good agreement between the experimental results and the model using a constant electrical conductivity is observed for the low-frequencies. Then the characteristic of the resistivity (obtained from the analysis of the loop diameter) and the capacitive effect related to permittivity could be deduced. As mentioned previously, the capacitances could be estimated through the slope of the plot of the imaginary part as a function of the frequency in Fig. 7c, 8c and 9c. However, at highfrequencies a discrepancy is observed between experiment and the model particularly in the real parts (Fig. 7b, 8b and 9b),
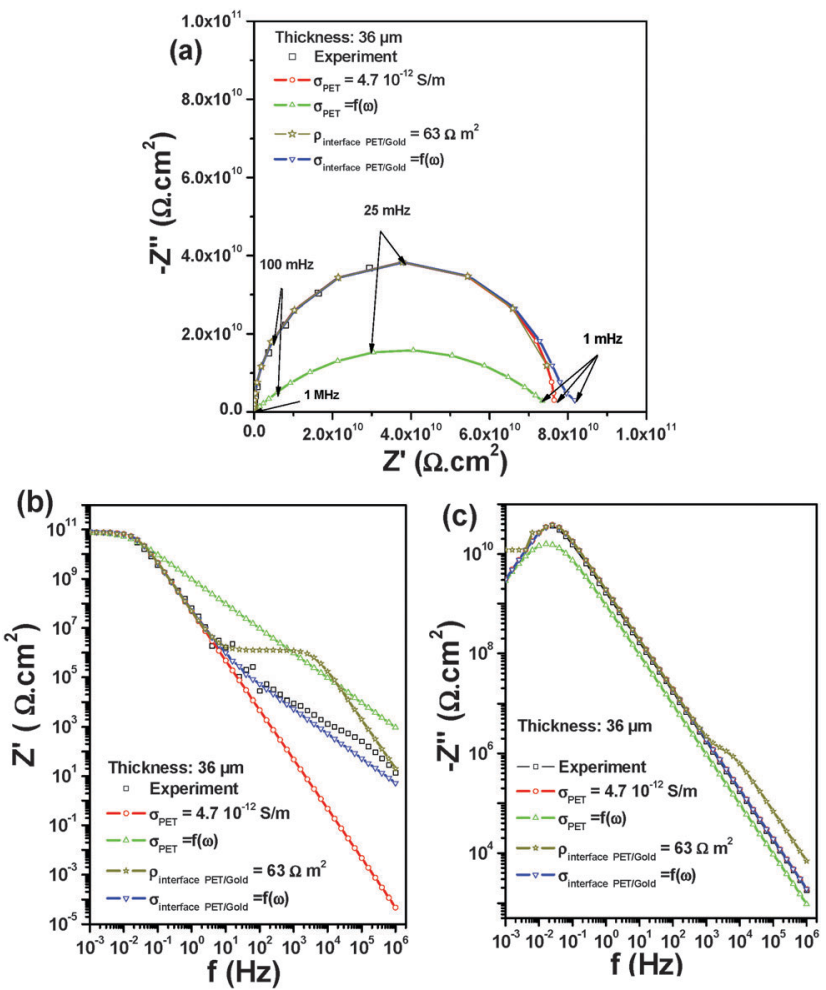

Fig. 7 Modeling by the finite elements of impedance diagrams measured on a PET disk of thickness $36 \mu \mathrm{m}$. (a) Nyquist diagram. (b) Real part with frequency. (c) Imaginary part with frequency.
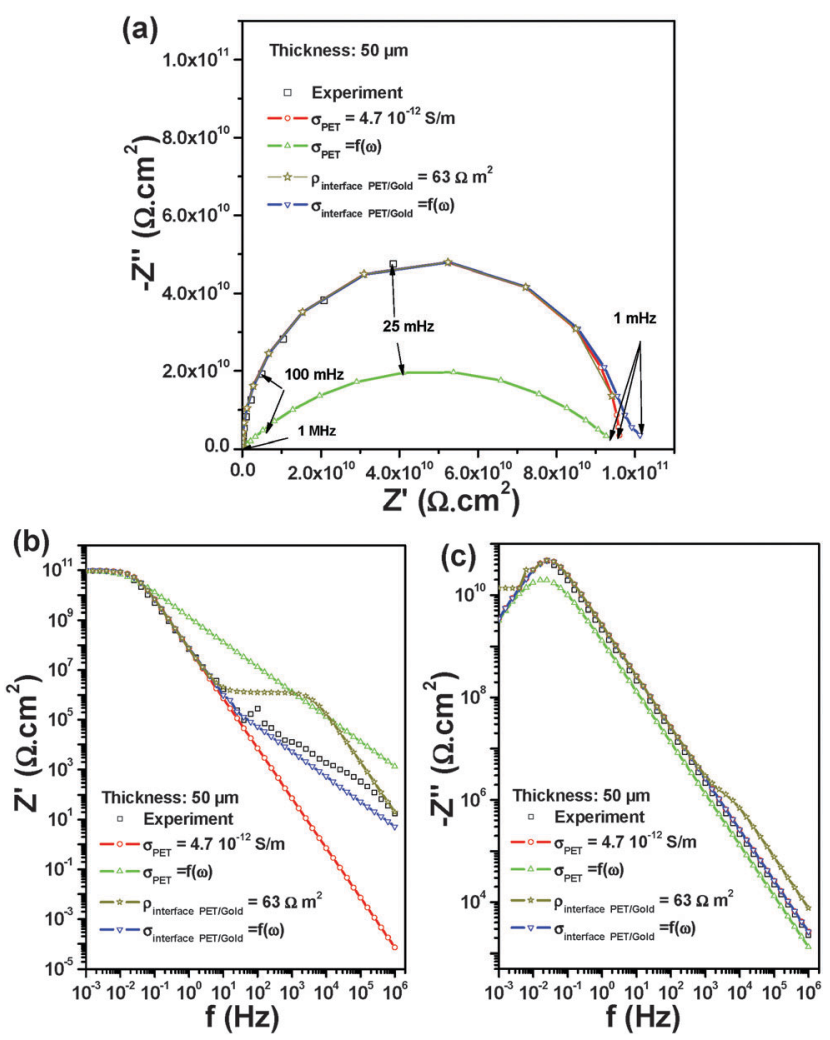

Fig. 8 Modeling by the finite elements of impedance diagrams measured on a PET disk of thickness $50 \mu \mathrm{m}$. (a) Nyquist diagram. (b) Real part with frequency. (c) Imaginary part with frequency. 
making the model less satisfactory. An analogy between the latter model and the previous electric equivalent circuit, $R_{\mathrm{PET}} / / C_{\mathrm{PET}}$, can be viewed. Indeed, the electrical conductivity depends on the PET resistivity while the relative permittivity is related to the geometric capacitance of the PET sample. Here again, the difference between the experimental points of the real part and those obtained by simulation can be ascribed to the non-uniform variation of PET resistivity at the interface. To account for such a phenomenon, a boundary condition on the distributed impedance was applied on the PET interface in order to take into account this interfacial resistance in the numerical calculation. The previous values found (Table 2) for the electric equivalent circuit were used with a contact resistance for testing it in the numerical calculation. A resistance of about $R_{\text {surface }}=63 \Omega \mathrm{m}^{2}$ allows a better fitting of the experimental results and it was fixed at the interface (Fig. 7-9). The result obtained by calculation confirms that the modelling impedance using an additional contribution such as a contact resistance at the PET/gold interface does not lead to a better representation of the real part of impedance. This model can be viewed as an equivalent description to the $R_{\text {cont }}+\left(R_{\mathrm{PET}} / / C_{\mathrm{PET}}\right)$ electrical equivalent circuit as mentioned above. As discussed previously, this also confirms that considering a constant electrical conductivity in the bulk PET and at the interfaces does not reflect the phenomena.

As a result, a frequency-dependent conductivity has to be taken into account in the polymeric material (see eqn (12)). ${ }^{27}$ The fitting procedure of our experimental results was performed assuming that the PET electrical conductivity leads to estimate the value of $A=3 \times 10^{-11}$, and $s=1$ (see Fig. 7-9). When the electric conductivity in the bulk PET is frequencydependent, the Nyquist diagram highlighted a flattened loop, which does not superpose with the experimental results. A significant difference is obtained for both the real and imaginary parts of impedance indicating that the difference between the theoretical and modelling data may be due to interfacial phenomena. The best fitting was obtained when a frequency-dependent conductivity is applied only at the interfaces while a constant conductivity is used for the bulk PET. Thus, for calculation it can be assumed that the electric resistance $\left(R_{\text {surface }}\right)$ and the electric conductivity $\left(\sigma_{\mathrm{S}}\right)$ are linked as follows:

$$
R_{\text {surface }}\left(\Omega \mathrm{m}^{2}\right)=\frac{d_{\mathrm{S}}}{\sigma_{\mathrm{S}}(\omega)_{\mathrm{PET}}}
$$

where $d_{\mathrm{S}}$ is the PET thickness along which the resistivity distribution occurs. The calculation for the fit procedure led to a thickness $d_{\mathrm{S}}$ of about $0.05 \mu \mathrm{m}$ along which the resistivity decreases from the interface.

To sum up, the experimental results are better simulated and fitted with the latter proposed model, in which special attention has been paid to account for the change in the resistive component of the measured impedance as a function of frequency. Therefore, the conductivity variation model used for the calculation using the finite element method is equivalent to the RD model on the PET membrane edges. In other words, this result indicates that the conductivity increases on a small
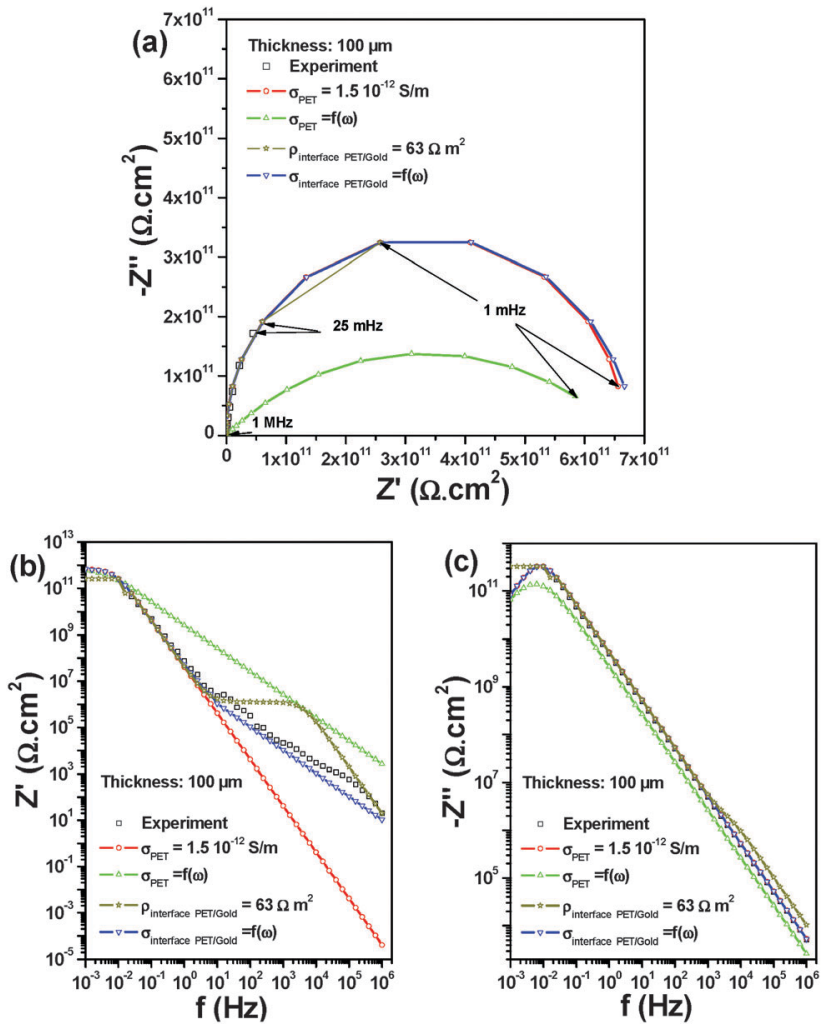

Fig. 9 Modeling by the finite elements of impedance diagrams measured on a PET disk of thickness $100 \mu \mathrm{m}$. (a) Nyquist diagram. (b) Real part with frequency. (c) Imaginary part with frequency.

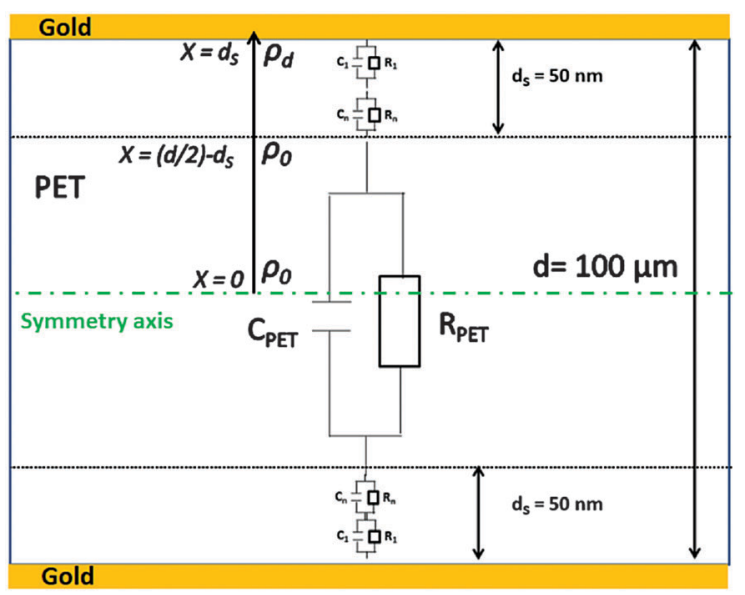

Fig. 10 Representation of the impedance model through the $100 \mu \mathrm{m}$-PET membrane. The bulk PET is represented by the $R_{\mathrm{PET}} / / C_{\mathrm{PET}}$ circuit while the $\mathrm{PET} /$ gold interface is represented by the resistivity distribution model.

distance localized in the vicinity of the PET/gold interfaces as illustrated in the scheme of Fig. 10.

Such a behaviour related to interfacial phenomena was previously highlighted by Neagu et $\mathrm{al}^{4}{ }^{4}$ Indeed, the high values of relative permittivity $\left(\varepsilon^{\prime}\right)$ observed at low frequencies were ascribed by "free" charges localized at the boundaries of conducting islet species or impurities in PET within the PET bulk, named interface polarization or Maxwell-Wagner-Sillars 
(MWS) polarization ${ }^{30}$ and at the PET sample/electrode interface named space-charge polarization. ${ }^{8}$ However, it can be assumed that space-charge polarization cannot occur at high frequencies due to the small applied AC period of the perturbation signal. Conversely, MWS polarization could occur at high frequencies because the transport of the charges over microscopic and mesoscopic dimensions will have sufficient time to build up at the boundaries of conducting islets. In our case, it can be assumed that the resistive behaviour observed can be understood in high frequencies mainly in the vicinity of the interface of PET which is here modelled by the RD model.

\section{Conclusions}

This study showed that the dielectric response of the PET membrane should not be considered as a simple system, such as $\left(R_{\mathrm{PET}} / / C_{\mathrm{PET}}\right)$, characterized only by electrical conductivity and relative permittivity. Indeed, strong interfacial behaviour was observed for the resistive component of the measured impedance at high frequencies. In the first approach using an electrical equivalent circuit, it was noted that the modelling results using a contact resistance in series with the simple dielectric representation as $R_{\text {cont }}+\left(R_{\mathrm{PET}} / / C_{\mathrm{PET}}\right)$ were not satisfactory while the CPE model was better for fitting the real part of the impedance but only within a restricted frequency range. In the second approach, the use of the analytical model of resistivity distribution shows that the interfacial behaviour was attributed to the decrease of resistivity localized at the interfaces of PET/gold. In the third approach using calculations with the finite element method has permitted us to confirm that the origin of the observed real part of impedance was ascribed to a conductivity increase at the interfaces of PET/gold along a small distance of $0.05 \mu \mathrm{m}$. These results are important for a better knowledge of the interfacial phenomena at the polymer/electrode interface mainly when microelectrodes were galvanically isolated in insulated polymer microdevices. Based on the conclusions of the present paper, work is under investigation to address the dielectric impedance modelling of the miniaturized PET channel onto which two planar microelectrodes are galvanically isolated with the flow channel.

\section{Acknowledgements}

This research was supported by the French research organism "Institut de Recherche pour le développement" (IRD).

\section{References}

1 W. Reddish, Trans. Faraday Soc., 1950, 46, 459.

2 A. S. Maxwell, I. M. Ward, F. Lauprêtre and L. Monnerie, Polymer, 1998, 39, 6835.

3 E. Neagu, P. Pissis and L. Apekis, J. Appl. Phys., 2000, 87, 2914.
4 E. Neagu, P. Pissis, L. Apekis and J. L. G. Ribelles, J. Phys. D: Appl. Phys., 1997, 30, 1551.

5 E. Neagu and R. Neagu, Electrets, 1994. (ISE 8), 8th International Symposium on, 1994, p. 528.

6 A. Thielen, J. Niezette, G. Feyder and J. Vanderschueren, J. Phys. Chem. Solids, 1996, 57, 1567.

7 J. Jackle, Rep. Prog. Phys., 1986, 49, 171.

8 A. Kyritsis, P. Pissis and J. Grammatikakis, J. Polym. Sci., Part B: Polym. Phys., 1995, 33, 1737.

9 M. Kechadi, J. Gamby, L. Chaal, H. Girault, B. Saidani and B. Tribollet, Electrochim. Acta, 2013, 105, 7.

10 S. Duval, M. Keddam, M. Sfaira, A. Srhiri and H. Takenouti, J. Electrochem. Soc., 2002, 149, B520.

11 Y.-j. Zhang, Y.-d. Huang and L. Wang, Solid State Ionics, 2006, 177, 65.

12 G. Schaumburg, Dielectrics Newsletter, Scientific Newsletter for Dielectric Spectroscopy, 1994, vol. 01, p. 4.

13 R. H. Boyd, Polymer, 1985, 26, 323.

14 E. Dargent, J. J. Santais, J. M. Saiter, J. Bayard and J. Grenet, J. Non-Cryst. Solids, 1994, 172-174(Part 2), 1062.

15 A. Schönhals and F. Kremer, in Broadband Dielectric Spectroscopy, ed. F. Kremer and A. Schönhals, Springer Berlin Heidelberg, 2003.

16 J. Mijovic, in Broadband Dielectric Spectroscopy, ed. F. Kremer and A. Schönhals, Springer Berlin Heidelberg, 2003.

17 M. G. Maitra, M. Sinha, A. K. Mukhopadhyay, T. R. Middya, U. De and S. Tarafdar, Solid State Ionics, 2007, 178, 167.

18 B. Hirschorn, M. E. Orazem, B. Tribollet, V. Vivier, I. Frateur and M. Musiani, J. Electrochem. Soc., 2010, 157, C458.

19 M. R. Shoar Abouzari, F. Berkemeier, G. Schmitz and D. Wilmer, Solid State Ionics, 2009, 180, 922.

20 B. Hirschorn, M. E. Orazem, B. Tribollet, V. Vivier, I. Frateur and M. Musiani, J. Electrochem. Soc., 2010, 157, C452.

21 M. Musiani, M. E. Orazem, N. Pébère, B. Tribollet and V. Vivier, J. Electrochem. Soc., 2011, 158, C424.

22 F. Sándor, Techniques de l'ingénieur Matières thermoplastiques: monographies 1997, base documentaire: TIB147DUO.

23 A. Mansingh, Bull. Mater. Sci., 1980, 2, 325.

24 L. X. He and S. C. Tjong, Eur. Phys. J. E: Soft Matter Biol. Phys., 2010, 32, 249.

25 F. Kremer and S. A. Różański, in Broadband Dielectric Spectroscopy, ed. F. Kremer and A. Schönhals, Springer Berlin Heidelberg, 2003.

26 D. P. Almond, G. K. Duncan and A. R. West, Solid State Ionics, 1983, 8, 159.

27 A. K. Jonscher, Nature, 1977, 267, 673.

28 H. Frohlich, W. Jackson and N. F. Mott, Theory of Dielectrics, Oxford University Press, London, 2nd edn, 1958.

29 N. E. Hill, W. E. Vaughan, A. H. Price and M. Davies, Dielectric Properties and Molecular Behavior, Van Nostrand Reinhold Compagny, London, 1969.

30 P. Hedvig, Dielectric Spectroscopy of Polymers, Akadémiai Kiadó, 1977. 